\title{
Cutaneous temperature and pressure pain threshold in individuals with knee osteoarthritis
}

\section{Fábio Marcon Alfieri ${ }^{1,2}$, Natália Cristina de Oliveira Vargas e Silva ${ }^{2}$, Artur César Aquino dos Santos ${ }^{1}$, Linamara Rizzo Battistella ${ }^{1,3}$}

${ }^{1}$ Clinical Research Center, Institute of Physical Medicine and Rehabilitation, Clinics Hospital (HCFMUSP), School of Medicine, University of São Paulo, São Paulo, Brazil

${ }^{2}$ Adventist University of São Paulo (UNASP), Brazil

${ }^{3}$ School of Medicine, University of São Paulo, São Paulo, Brazil

\begin{abstract}
Introduction: Osteoarthritis is a common disease in which skin temperature may be included among the pathophysiological factors. Thermography allows the mapping of cutaneous temperature and may be employed in the investigation of osteoarthritis.

Objectives: To evaluate cutaneous temperature of the lower limbs, as well as to verify its association with pressure pain thresholds in individuals with knee osteoarthritis.

Material and methods: This case series study was conducted with individuals of both genders aged 48-77 years with unilateral knee osteoarthritis. Volunteers underwent thermographic evaluation by an infrared sensor (FLIR T650SC). The anterior region of the thigh and leg and the knee area were evaluated. Pressure pain thresholds (PPT) were evaluated by algometry (Pain Diagnostics, Great Neck, USA) at the vastus medialis, vastus lateralis, rectus femoris, and patellar tendon. Data analysis was conducted with the statistical package SPSS v.24 for Windows. Comparisons between affected and unaffected sides were made by paired Student's $t$-test or the Mann-Whitney $U$ test, and associations between variables were assessed by Pearson or Spearman's correlation coefficient. In all cases, the significance level was set at $p \leq 0.05$.

Results: Eleven volunteers ( $63.1 \pm 9.5$ years) participated in this study. When comparing cutaneous temperature, only the region of the knee showed a significant difference between sides $(p=0.02)$. There were no differences between affected and unaffected knees regarding pain tolerance (PPT) at all sites evaluated. There were also no significant associations between the study variables.

Conclusions: Individuals with knee osteoarthritis presented a higher temperature of the affected knee, but this was not associated with pressure pain thresholds.
\end{abstract}

Key words: osteoarthritis, body temperature, thermography, pain measurement.

\section{Introduction}

Considered to be the most common joint disease worldwide, osteoarthritis (OA) affects $50 \%$ or more of the elderly population and is present in about $10 \%$ of the knees of women aged over 60 years [1-5]. Osteoarthritis is a major cause of pain and disability to patients [1-5].

Imaging examinations such as X-ray, computed tomography and magnetic resonance may provide information about the articular structure [6]. Other evaluations are useful to evaluate disability, including specific questionnaires such as the Western Ontario and McMaster Universities Osteoarthritis Index (WOMAC) [7]. Imaging modalities such as scintigraphy and magnetic resonance allow functional physiological information. Thermography is within this functional imaging category [6]. It is an examination that assesses skin surface temperature through infrared radiation, the most efficient method for 
the study of cutaneous temperature distribution [8-11]. The use of this technique is interesting for being completely painless, fast and non-invasive, besides having no contraindications or side effects.

Cutaneous temperature is a significant indicator of physiological dysfunctions, since temperature is an indicator of health status [9-11].

As thermography allows the mapping of possible areas of ischemic tissue or with increased temperature [12] it is recommended that it be used in the investigation of $\mathrm{OA}[11-13]$.

The study by Varjú et al. [14] found that cutaneous temperature of the joints varied with the severity of OA. Affected joints were warmer than usual at the beginning of the course of OA. As the disease progressed and symptoms worsened, surface temperature of the joints seemed to decrease, but this study was conducted in individuals with $\mathrm{OA}$ in the hand and fingers. Other studies have already shown a good correlation between the increase in temperature and important radiographic changes in individuals with knee OA $[6,15]$.

Regarding the association between pain and cutaneous temperature, a study observed an association between myofascial trigger points of masticatory muscles with algometry and thermography, and verified a positive and moderate correlation. The authors verified that the higher the applied force was, the higher was the local temperature [16].

Knee temperature does not appear to be a predictor of pain in the knees of elderly individuals. However, the author of this study evaluated pain by questionnaires (Knee Pain Scale and WOMAC pain subscale) [17]. As the mechanisms of pain in OA are still not fully established and understood $[3,4]$, precise methods such as thermography and the objective evaluation of pain through pressure pain thresholds (a technique already employed by previous studies) $[18,19]$ can bring information about the relationship between pain and cutaneous temperature in this disease. Although thermography is a reliable diagnostic tool to detect quantifiable cutaneous temperature patterns in patients with $\mathrm{OA}$ [18], a recent study highlights the fact that current scientific literature is still scarce about the employment of this technique in OA [19].

Therefore, the aim of this study was to evaluate cutaneous temperature of the lower limbs, and to verify its association with pressure pain thresholds in individuals with knee osteoarthritis.

\section{Material and methods}

This case series study was conducted at the Institute of Physical Medicine and Rehabilitation (IMREA, in its
Portuguese acronym) of the Clinicas Hospital of the University of São Paulo. Volunteers gave written informed consent to participate in this study, which was approved by the local Research Ethics Committee, protocol number 1.455 .745 .

Individuals of both genders aged 48-77 years with unilateral knee OA were recruited to participate in the study. At the time of data collection, they were being treated at IMREA.

Persons with severe cognitive disorders that prevented the understanding of the study procedures, those who presented fever due to any infection, and those with a history of vascular abnormalities or knee arthroplasty were excluded from the sample.

Volunteers underwent an anamnesis including age, weight, height, affected knee, disease duration, physical disability measured by the WOMAC questionnaire [7], and pain intensity measured by the visual analog scale (VAS) [20]. Before further evaluations, volunteers were instructed not to consume alcohol, caffeine or tobacco, not to use any type of cream or moisturizer, and not to perform strenuous physical activity in the 24 hours that preceded data collection. Once these requirements were met, on the day of the evaluation each volunteer was instructed to wear clothing that would allow the exposure of the lower limbs, so that they could be brought into thermal equilibrium with the temperature of the examination room $\left(21.3 \pm 0.4^{\circ} \mathrm{C}\right.$, humidity of $\left.63.4 \pm 2.7 \%\right)$.

Subjects remained standing 2 meters away from the infrared sensor, and $0.4 \mathrm{~m}$ from the wall. Thermal images were captured by FLIR T650SC infrared sensor, with thermal sensitivity of $0.03^{\circ} \mathrm{C}$, resolution of $640 \times 480$ pixels, image frequency of $30 \mathrm{~Hz}$, spatial resolution of $0.69 \mathrm{mrad}$, temperature range verification of $-40^{\circ} \mathrm{C}$ to $2000^{\circ} \mathrm{C}$, and accuracy of $1^{\circ} \mathrm{C}$ or $\pm 1 \%$ of reading (limited temperature range). Images were taken assuming the emissivity of the skin of 0.98. Subjects were instructed not to perform arm or leg movements, or to scratch any region of the body before or during the procedure. The procedure of evaluation of cutaneous temperature obtained by thermography followed the criteria adopted and recommended by the literature [11, 13, 21].

Thermal images were analyzed by FLIR Tools software. The average temperature was measured in degrees Celsius in each region of interest (ROI). The ROIs were rectangles determined by anatomical points, namely: thigh $(5 \mathrm{~cm}$ above the superior border of the patella and $5 \mathrm{~cm}$ below the inguinal line) and leg $(5 \mathrm{~cm}$ below the inferior border of the patella and $10 \mathrm{~cm}$ above the malleolus) [21]. The knee region was also assessed by a circle involving the entire anterior knee, between the other two ROls. All evaluations were conducted in the anterior view as shown in Figure 1. 


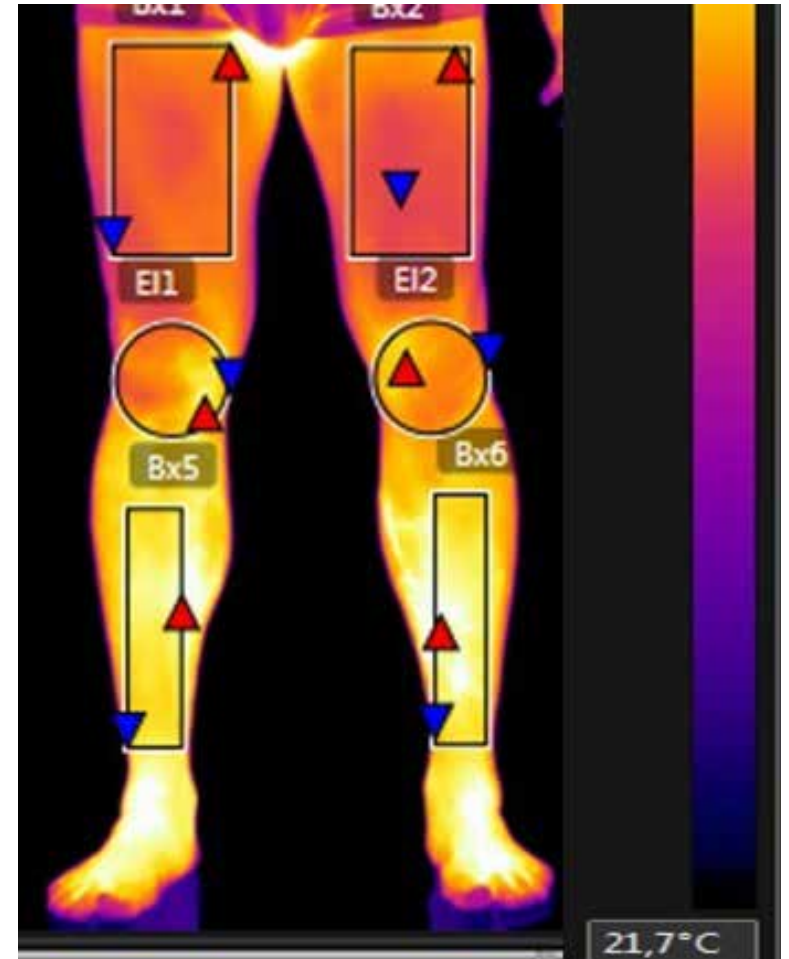

Fig. 1. Thermographic image showing the region of interest (ROI). Thigh: region defined by the rectangles on the top. Leg: limited by the rectangles on the bottom. Knee: area defined by circles. The image was taken at the Thermography Laboratory of the Institute of Physical Medicine of the Clinicas Hospital, University of São Paulo (Brazil).

After this, pressure pain thresholds (PPT) were evaluated; PPT were assessed by a pressure algometer (Pain Diagnostics, Great Neck, USA). A constant pressure was applied at $1 \mathrm{~kg} / \mathrm{s}$ until the patient reported the onset of pain or discomfort. Pressure was applied perpendicularly to the skin surface. The reading was expressed in $\mathrm{kg} / \mathrm{cm}^{2}$.

Table I. Characteristics of study participants

\begin{tabular}{|lc|}
\hline Variables & \\
\hline$N$ & 11 \\
\hline Age (years) & $63.1 \pm 9.5$ \\
\hline BMI $\left(\mathrm{kg} / \mathrm{m}^{2}\right)$ & $28.7 \pm 4.0$ \\
\hline Affected side (R/L) & $8 / 3$ \\
\hline Time of pain (months) & $57.8 \pm 40.4$ \\
\hline VAS (cm) & $3.6 \pm 3.5$ \\
\hline WOMAC $(\%)$ & $31.0 \pm 19.21$ \\
\hline
\end{tabular}

$B M I$ - body mass index, $R$ - right, $L$ - left, VAS - visual analog scale, WOMAC - Western Ontario and MCMaster Universities Osteoarthritis Index.

Data are expressed as means standard \pm deviations or absolute values.
During the assessment, subjects were instructed to say "stop" as soon as the pressure sensation became painful, and that pressure level was registered. Sites assessed by algometry were: vastus medialis, vastus lateralis, rectus femoris and patellar tendon. These points have already been assessed and described by previous studies [22, 23].

\section{Data analysis}

Data analysis was conducted with the statistical package SPSS v.24 for Windows. Data are presented as means \pm standard deviations. Normality of data was assessed by the Kolmogorov-Smirnov method. Comparisons between affected and unaffected sides were made by paired Student's $t$-test or the Mann-Whitney $U$ test, and associations between variables were evaluated by Pearson or Spearman's correlation coefficient, and classified as follows: 0.0 to 0.19 - very weak correlation; 0.2 a 0.39 - weak correlation; 0.4 to 0.69 - moderate correlation; 0.7 to 0.89 - strong correlation; 0.9 a 1.0 very strong correlation. In all cases, the significance level was set at $p \leq 0.05$.

\section{Results}

Seventeen individuals with knee OA were invited to participate in the study. Out of them, 6 were excluded from the analysis due to having a prosthesis in one knee $(n=1)$, presenting a history of thrombosis $(n=1)$ or having bilateral symptoms $(n=4)$. Baseline characteristics of the remaining 11 patients are shown in Table I.

The temperature comparison of both affected and unaffected knees revealed a statistically significant difference of temperature between the two sides. This finding is shown in Table II. All other ROI comparisons failed to detect significant differences.

Table II. Comparisons between affected and unaffected sides

\begin{tabular}{|lccc|}
\hline Variables & $\begin{array}{c}\text { Affected } \\
\text { side }\end{array}$ & $\begin{array}{c}\text { Unaffected } \\
\text { side }\end{array}$ & $P$ \\
\hline Temperature $\left({ }^{\circ} \mathrm{C}\right)$ & & & \\
\hline Thigh & $29.9 \pm 1.1$ & $29.9 \pm 1.2$ & 0.65 \\
\hline Knee & $29.5 \pm 1.4$ & $29.2 \pm 1.2$ & 0.03 \\
\hline Leg & $31.4 \pm 1.0$ & $31.2 \pm 1.0$ & 0.19 \\
\hline Pressure pain threshold $\left(\mathrm{kg} / \mathrm{cm}^{2}\right)$ & & \\
\hline Vastus medialis & $4.5 \pm 2.4$ & $6.1 \pm 3.2$ & 0.17 \\
\hline Vastus lateralis & $6.3 \pm 2.9$ & $6.1 \pm 2.1$ & 0.61 \\
\hline Rectus femoris & $6.3 \pm 2.7$ & $6.2 \pm 2.9$ & 0.87 \\
\hline Patellar tendon & $6.7 \pm 2.8$ & $6.3 \pm 1.9$ & 0.65 \\
\hline
\end{tabular}

Data are expressed as means standard \pm deviations. 
There were weak and very weak correlations between the temperature of the affected knee and the other study variables, but they were not significant (Table III).

\section{Discussion}

In this case series study, we found a difference in the temperature of the knee ROI between affected and unaffected sides of individuals with knee OA. The non-significant weak and very weak associations between knee temperature and pain intensity, pressure pain tolerance threshold and disability (WOMAC) indicate that cutaneous temperature of the knee seems not to be associated with pain and disability.

Normal patterns of cutaneous temperature are complex; thus, sensorial investigation associated with thermography and a good clinical evaluation should be considered [24]. Hence, our study evaluated the association of thermography and clinical assessments of pain, function, and pain pressure threshold of patients with knee osteoarthritis.

Regarding the sensorial evaluation of PPT, it was expected that the affected side would present a lower pain threshold. Nevertheless, no differences were observed in pain tolerance when comparing the knee affected by $\mathrm{OA}$ and the heathy contralateral knee. This was probably due to the central sensitization mechanism that this disease brings about over time [3, 5, 23].

Another interesting fact is that although pain was considered moderate at the time of data collection (mean of 3.6 in VAS), knee temperature was statistically significantly different between affected and unaffected sides. Nonetheless, the difference of $0.3^{\circ} \mathrm{C}$ is not relevant. Even though healthy individuals tend to present small differences between body hemispheres (close to $0^{\circ} \mathrm{C}[25]$ ), there was previously reported an average mean difference of $0.4^{\circ} \pm 0.3^{\circ} \mathrm{C}$ [9] between right and left sides of healthy individuals, a value close to the difference observed in the present study.

A recent study [19] aimed at verifying cutaneous temperature of lower limbs in persons with knee OA and healthy individual observed that male patients tend to present a higher temperature in the thigh, and lower in the knee when compared to the control group. No differences were observed among women. When comparing right and left sides, the authors observed significant differences in the popliteal region (higher temperature on the left side) in both patients and controls, a quite unexpected result. In the present study, where patients with unilateral knee OA were included, cutaneous temperature of the anterior joint region of the knee was the only variable presenting a significant difference between affected and unaffected knees. This must have been due to an increase in local catabolic activity in the affected knee, as highlighted by Brito et al. [19].
Table III. Associations between study variables

\begin{tabular}{|lcc|}
\hline Temperature & $r$ & $p$ \\
\hline Temperature $($ knee ROI) $\times$ VAS & 0.20 & 0.54 \\
\hline Temperature $($ knee ROI) $\times$ WOMAC & 0.24 & 0.46 \\
\hline Temperature $($ knee ROI) $\times$ PPT VM & 0.25 & 0.45 \\
\hline Temperature $($ knee ROI) $\times$ PPT VL & -0.34 & 0.29 \\
\hline Temperature $($ knee ROI) $\times$ PPT RF & -0.05 & 0.86 \\
\hline Temperature $($ knee ROI) $\times$ PPT PT & -0.03 & 0.91 \\
\hline
\end{tabular}

$R O I$-region of interest, VAS - visual analog scale, WOMAC - Western Ontario and McMaster Universities Osteoarthritis Index, PPT - pressure pain threshold, VM - vastus medialis, VL - vastus lateralis, $R F$ - rectus femoris, $P T$ - patellar tendon.

Regarding disability (assessed by WOMAC), the present data did not reveal an association between this variable and knee temperature. This suggests that, in this group of individuals with knee OA, cutaneous temperature of the knee may not be associated with disability.

Corroborating the study by Tsai et al. [17], no significant associations between knee temperature and any measure of knee pain were found in the present study. Even the objective assessment of PPT was not significantly associated with knee temperature in individuals with knee OA.

The results presented herein are limited and must be considered with caution. This was a pilot study of a case series; therefore a small sample was included, and patients were classified for OA severity. Studies with larger samples that associate thermography with other objective assessments in randomized clinical trials are encouraged.

\section{Conclusions}

Individuals with unilateral knee osteoarthritis and with moderate pain had a significant difference in the joint temperature comparing with OA unaffected knee. However, no significant associations between knee temperature and pain, pressure pain thresholds or disability were observed.

The authors declare no conflict of interest.

\section{References}

1. Staud R. Evidence for shared pain mechanisms in osteoarthritis, low back pain, and fibromyalgia. Curr Rheumatol Rep 2011; 13: 513-520, DOI: 10.1007/s11926-011-0206-6.

2. Alkan BM, Fidan F, Tosun A, Ardıçoğlu O. Quality of life and self-reported disability in patients with knee osteoarthritis. Mod Rheumatol 2014; 24: 166-171, DOI: 10.3109/14397595.2013.854046.

3. Sofat N, Ejindu V, Kiely P. What makes osteoarthritis painful? The evidence for local and central pain processing. Rheumatology (Oxford) 2011; 50: 2157-2165, DOI: 10.1093/rheumatology/ ker283. 
4. Hunter DJ, McDougall JJ, Keefe FJ. The symptoms of osteoarthritis and the genesis of pain. Rheum Dis Clin North Am 2008; 34: 623-643, DOI: 10.1016/j.rdc.2008.05.004.

5. Salaffi F, Ciapetti A, Carotti M. The sources of pain in osteoarthritis: a pathophysiological review. Reumatismo 2014; 66: 57-71, DOI: 10.4081/reumatismo.2014.766.

6. Denoble AE, Hall N, Pieper CF, Kraus VB. Patellar skin surface temperature by thermography reflects knee osteoarthritis severity. Clin Med Insights Arthritis Musculoskelet Disord 2010; 3: 69-75, DOI: 10.4137/CMAMD.S5916.

7. Bellamy N, Campbell J, Stevens J, et al. Validation study of a computerized version of the Western Ontario and McMaster Universities VA3.0 Osteoarthritis Index. J Rheumatol 1997; 24: 2413-2415.

8. Jiang LJ, Ng EY, Yeo AC, et al. A perspective on medical infrared imaging. J Med Eng Technol 2005; 29: 257-267, DOI: 10.1080/ 03091900512331333158.

9. Vardasca R, Ring EFJ, Plassmann P, Jones C. Thermal symmetry of the upper and lower extremities in healthy subjects. Thermol Int 2012; 22: 53-60.

10. Lahiri BB, Bagavathiappan S, Jayakumar T, Philip J. Medical applications of infrared thermography: A review. Infrared Phys Technol 2012; 55: 221-235, DOI: 10.1016/j.infrared.2012. 03.007.

11. Fernández-Cuevas I, Bouzas Marins JC, Arnáiz Lastras J, et al. Classification of factors influencing the use of infrared thermography in humans: A review. Infrared Phys Technol 2015; 71: 28-55, DOI: 10.1016/j.infrared.2015.02.007.

12. Szentkuti A, Kavanagh HS, Grazio S. Infrared thermography and image analysis for biomedical use. Periodicum Biologorum 2011; 113: 385-392.

13. Ring EF, Ammer K. Infrared thermal imaging in medicine. Physiol Meas 2012; 33: R33-46, DOI: 10.1088/0967-3334/33/3/R33.

14. Varjú G, Pieper CF, Renner JB, Kraus VB. Assessment of hand osteoarthritis: correlation between thermographic and radiographic methods. Rheumatology (Oxford) 2004; 43: 915-919, DOI: 10.1093/rheumatology/keh204.

15. Warashina $\mathrm{H}$, Hasegawa $\mathrm{Y}$, Tsuchiya $\mathrm{H}$, et a $\mathrm{l}$. Clinical, radiographic, and thermographic assessment of osteoarthritis in the knee joints. Ann Rheum Dis 2002; 61: 852-854, DOI: 10.1136/ ard.61.9.852.

16. Haddad DS, Brioschi ML, Arita ES. Thermographic and clinical correlation of myofascial trigger points in the masticatory muscles. Dentomaxillofac Radiol 2012; 41: 621-629, DOI: $10.1259 / \mathrm{dmfr} / 98504520$.

17. Tsai PF, Richards K, Tatom I. The association between knee temperature and pain in elders with osteoarthritis of the knee: a pilot study. J Adv Nurs 2003; 42: 373-381, DOI: 10.1046/j.1365-2648.2003.02629.x.

18. Arfaoui A, Bouzid MA, Pron $\mathrm{H}$, et al. Application of infrared thermography as a diagnostic tool of knee osteoarthritis. J Therm Sci Technol 2012; 7: 227-235.

19. Brito CJ, Miarka B, Garcia-Pastor T, et al. Osteoarthritis subjects have differentiated lower extremity thermal skin response after the concurrent acute training session. J Therm Anal Calorim 2020, DOI: https//doi.org/10.1007/s10973-020-09827-0.

20. Carlsson AM. Assessment of chronic pain. I. Aspects of the reliability and validity of the visual analogue scale. Pain 1983; 16: 87-101, DOI: 10.1016/0304-3959(83)90088-x.

21. Marins JC, Fernandes AA, Cano SP, et al. Thermal body patterns for healthy Brazilian adults (male and female). J Therm Biol 2014; 42: 1-8, DOI: 10.1016/j.jtherbio.2014.02.020.

22. Imamura M, Ezquerro F, Marcon Alfieri F, et al. Serum levels of proinflammatory cytokines in painful knee osteoarthritis and sensitization. Int J Inflam 2015; 2015: 329792, DOI: 10.1155/2015/329792.

23. Imamura M, Imamura ST, Kaziyama HH, et al. Impact of nervous system hyperalgesia on pain, disability, and quality of life in patients with knee osteoarthritis: a controlled analysis. Arthritis Rheum 2008; 59: 1424-1431, DOI: 10.1002/art.24120.

24. Roy RA, Boucher JP, Comtois AS. Comparison of paraspinal cutaneous temperature measurements between subjects with and without chronic low back pain. J Manipulative Physiol Ther 2013; 36: 44-50, DOI: 10.1016/j.jmpt.2012.12.002.

25. Gross MT, Schuch CP, Huber E, et al. Method for quantifying assessment of contact thermography: effect of extremity dominance on temperature distribution patterns. J Orthop Sports Phys Ther 1989; 10: 412-417, DOI: 10.2519/jospt.1989.10.10.412. 